\title{
A study on well-being, locus of control, gender and sports
}

\author{
Ankita Agarwal ${ }^{1}$, Chetna Mulani ${ }^{2}$, Shrushti Mehta ${ }^{3}$, Mandeep Kaur ${ }^{4}$, Chitra Munshi ${ }^{5}$ \\ ${ }^{1-4}$ TYBA Psychology Students, ${ }^{5}$ Head of Department \\ Department of Psychology, L. S. Raheja College, Mumbai. \\ Corresponding author: Dr. Chitra Munshi \\ Email-chitram3@yahoo.co.in
}

\begin{abstract}
Background: The purpose of the study is to investigate the general well-being in students who play sports at a competitive level and students who don't play sports at a competitive level of the age group 1622 years.

Methods: The sample consisted of 160 participants. 80 participants played sports at a competitive level (out of which 40 were males and 40 were females) and 80 participants did not play sports at a competitive level (out of which 40 were males and 40 were females). Method of purposive sampling and convenience sampling was used for inclusion of participants.

Results: The difference of general well-being between internal and external locus of control was significant at $\mathrm{F}=11.58, \mathrm{p}<0.000852$ and the difference of general well-being between male and female was significant at $\mathrm{F}=9.46, \mathrm{p}<0.002491$.

Conclusion: Sport in the researcher's sample, did not have major impact on the general well-being of students. The researcher's study, was in line with previous researches, saying that gender has a major impact on general well-being of an individual, this is because of several psycho-social factors.
\end{abstract}

Keywords: Well being, Locus of Control, Males, Females, Sports, Non-Sports.

(Paper received $-1^{\text {st }}$ January 2018, Peer review completed $-12^{\text {th }}$ January 2018)

(Accepted $-16^{\text {th }}$ January 2018)

\section{INTRODUCTION}

Mental health and sports go hand in hand. What started in 1938 by the Chicago Club owner, hiring of the father of sports psychology Coleman Roberts Griffith, has now turned into one of the leading sectors of the commercial world with institutes like Premier Sport Psychology, Mind of the Athlete, KPEX Consulting etc. For people who participate, sports doesn't end with the last tick of the scoreboard clock, sports persist inside them as memories and feelings and all this gets translated into a generalised conception of themselves, it reinforces their self concept in areas beyond sport-related part of their self definition.

Well-being or wellness is a general term for the condition of a group and/or an individual. A high level of well-being means in some sense the individual or group's condition is positive. Mental well-being describes your state of mind, how you are feeling and how well you can cope with day-to-day life situations.

General well-being of the individual can often be determined by the participation of the individual in various social events including competitive sports. In area of competitive sports, individuals face various situations which lead to stress and anxiety which in turn affects their behavior and thought pattern.

Locus of control is a psychological concept that refers to how strongly people believe they have control over the situations and experiences that affect their lives. According to psychologist Julian Rotter, who formulated the concept in the 1950s, the locus of control is a dimension of personality, it helps explain one's traits and behavior. People who base their success on their own work and believe they control their 
life have an internal locus of control. In contrast, people who attribute their success or failure to outside influences have an external locus of control [1].

A study was done on role of Locus of Control and Gender on Psychological Well being among Youth Athletes [2]. The sample size was of 220 undergraduate athletes in Kenya. The researchers found that people with internal locus of control have a higher psychological well being than people with external locus of control. Also, male athletes have a higher psychological well-being than female athletes.

A study was done on the relationship of sport participation and adjustment, the measure used was locus of control, they compared male and female participants and nonparticipants. The results indicated that a higher percentage of female sport participants had an internal locus of control and higher percentage of male non participants had an external locus of control. [3]

Another study was done to determine whether young black adults who participate in sport differ in psychological well-being and psychosocial development from those who do not participate actively in sport. An availability sample of 293 students between the age of 20 and 35 years was used. Sport participants manifested higher levels of wellness on various facets of psychological well-being and psychosocial development. [4]

An international study on 12 European countries was done to explore the impact of sporting success and sporting events on self-reported life satisfaction. The conclusion derived was that national sporting success can lead to an increased sense of life satisfaction, but the effect is not a very powerful one, the results did not generally support the view that there are systematically significant and positive anticipatory or postevent legacy effects with respect to measured well-being [5].

A recent study aimed to understand the relation between personality traits and sport performance. Personality traits consisted of five dimensions (extraversion, neuroticism, openness, agreeableness, and conscientiousness). The population of the study consisted of 229 non elite football and futsal players. The results showed that among personality components only Conscientiousness had positive significant correlation with players performance [6].

Statement of problem: To investigate the role of locus of control and gender on general well being for sport and non sport participants. Sports are an important aspect in the life of students, a lot of Indian Sports men and women have now succeeded in various worldwide competitions. The researchers were intrigued to find out whether students who played sports at a competitive level have a higher well being as they are exposed to a certain kind of physical fitness. Keeping in mind the influence of social factors and the dominance of men in the field of sport, gender was included as a variable in the study. Having experienced certain situations in their sports career such as leadership, performance pressure, failure, stressful competition, etc., it is speculated that sports people have an internal locus of control as they become responsible for their own actions.

\section{METHODOLOGY}

\section{Sample:}

The researchers have used a convenience, purposive sampling.

160 people participated in this study. The sample was equally divided into 4 groups i.e. 40 each in Male sports, female sports, male non sports and female non sports. There were sub categories that further divided the four groups based on the locus of control of the student, there were 123 participants with an internal locus of control and 37 participants with an external locus of control.

The participants were literate and the age group for the sample was 16-22 years mean age being 19.3 years. The sampling technique used was convenience and purposive sampling. Students below the age of 16 and above the age of 22 were excluded.

\section{Procedure}

Data was divided into different categories for comparison. The score on general well-being scale was compared on the basis of 3 dimensions of gender, sport participation and locus of control. Two scales were used and data was analyzed. In Levenson's LOC [7] scale, the highest score out of the three dimensions was considered and the score of powerful other and chance was taken as external LOC score. VASAAR 
was used to conduct the $2 \times 2 \times 2$ ANOVA, to compare the general well-being of participants, segregated on the bases of sport participation, gender and locus of control.

\section{Tools}

\section{General Well-being Scale:}

The Psychological General Well-Being Index (PGWBI) is a measure of the level of subjective psychological well-being. In detail, it assesses self-representations of intra-personal affective states reflecting a sense of subjective well-being or distress and captures what we could call a subjective perception of well-being. In the 1960s, Harold Dupuy, psychologist at the National Center for Health Statistics, developed his Psychological General Well-Being (PGWB) Schedule. He devised a questionnaire of 68 items to measure the psychological distress of the American population, later there was a drastic reduction in the number of items to ensure applicability and acceptability of the questionnaire on a large scale, and only 18 items were chosen to be effectively used in the NHANES in 1971. Subsequently, the collaboration between Harold Dupuy and the RAND Corporation led to a revision, adaptation, and validation of the final PGWB Schedule and its transformation to its final form, the PGWB Index.

\section{Locus of control (LOC) scale}

LOC is a likert-type, summative scale used to measure the attitudes of people. Its multi-dimensional, consisting of 3 dimensions namely internal, chance and powerful others. Scores on all 3 dimensions can be low or high since these 3 dimensions are independent of each other. The scale ranges from strongly disagree to strongly agree (-3 to 3 ). LOC is a widely used, psychometrically sound psychological tool. For the purposes of this study, participants with highest score on internal scale were categorized as internal LOC participants and participants who got high on the powerful others or chance scales were categorized as external LOC participants.

\section{Independent Variable}

Sports Participation at a competitive level (participation and no participation): Sports participants can be operationally defined as people who have played or are playing outdoor or indoor sports at a competitive level.

Sport non participants can be operationally defined as people who do not play outdoor or indoor sports at a competitive level.

Locus of control: Internal LOC can be operationally defined as scoring highest on internal dimension on LOC scale

External LOC can be operationally defined as scoring higher on powerful others or chance scales.

Gender - male and female

Dependent Variable:

General Well-Being score on General Well-Being Scale.

Design - The research design was a survey research design, using a self-report questionnaire.

\section{Hypotheses}

There are three hypotheses in this study, they are:

1. General well-being is higher in students who play sports at a competitive level than people who don't play sports at a competitive level.

2. Gender plays a significant role on the general well-being of people

3. Students having a internal locus of control have a higher general well being than students having a external locus of control. 
The result of this study is as follows

\section{RESULTS}

- The difference of general well-being between sports and non sports participation was not significant. $\mathrm{F}=0.01, \mathrm{p}<0.920476$.

- The difference of general well-being between internal and external locus of control was significant at $\mathrm{F}=11.58, \mathrm{p}<0.000852$.

- The difference of general well-being between male and female was significant at $\mathrm{F}=9.46$, $\mathrm{p}<0.002491$.

- The interaction between sports participation and locus of control was not significant. $F=1.31$, $\mathrm{p}<0.254194$.

- The interaction between sports participation and gender was not significant. $F=0, p<1$.

- The interaction between locus of control and gender was not significant. $F=2.08, p<0.151298$.

- The interaction between sports participation, locus of control and gender is not significant. $F=0.42$, $\mathrm{p}<0.517915$.

\section{Count of MALE SPORTS}

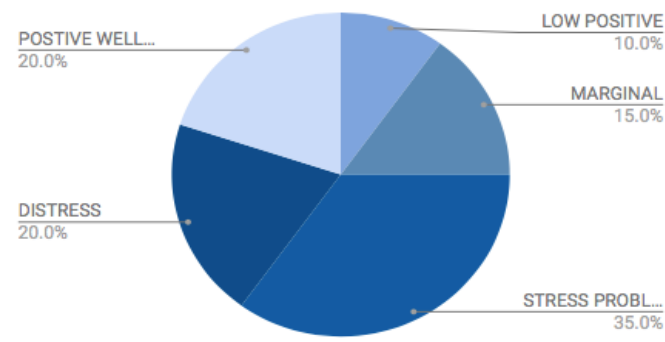

Count of MALE NON SPORTS

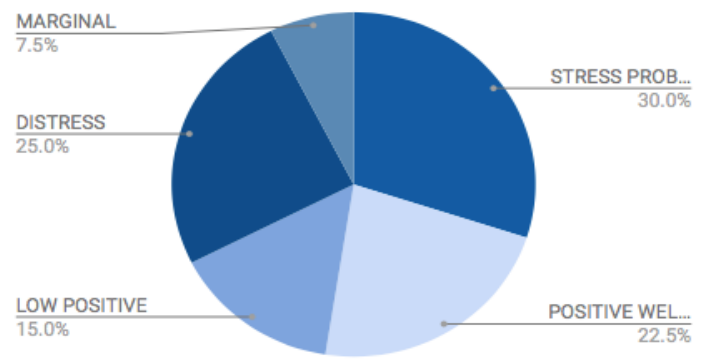

Count of FEMALE SPORTS

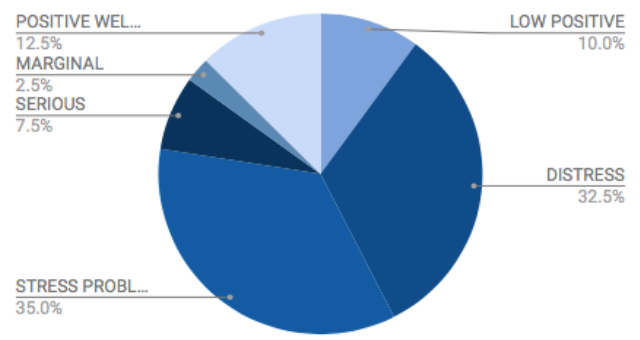

Count of FEMALE NON SPORTS

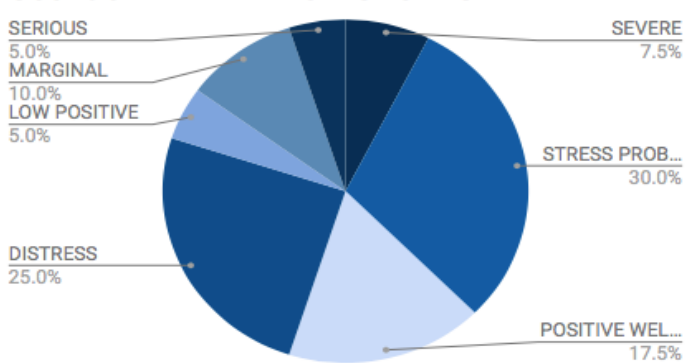

\section{DISCUSSION}

In Hypothesis 1, General well-being is higher in students who play sports at a competitive level than people who don't play sports at a competitive level, the results were not significant because $\mathrm{F}=0.01$, $\mathrm{p}<0.920476$.

In Hypothesis 2, Gender plays a significant role on the general well-being of people, the results were significant as $\mathrm{F}=9.46, \mathrm{p}<0.002491$.

In Hypothesis 3,Students having a internal locus of control have a higher general well being than students having a external locus of control, the results were significant as $F=11.58, \mathrm{p}<0.000852$.

With reference to the results of the study there is no significant difference in the general well being between students who participate in sports at a competitive level and students who do not participate in sports at a competitive level. There are multiple reasons for this including the social influences of the Indian society where not all sports are given equal importance and status. For example, cricket is given more importance 
than other sports, the only time cricket shares the spotlight with another sporting event is during the Olympics. It is then, once every four years, that the media and public collectively express their outrage at the failure of the sports system in the country and blame the government for the poor performance of athletes at the games. [7]

A greater significance is seen between internal locus of control and external locus of control. The mean general well being of internal locus of control is 66.6829 and of external locus of control is 56.8919 . The results are so because having an internal locus of control itself means that people consider themselves responsible for their actions hence, being more confident and successful which in turn leads to higher general well being.

A similar study was conducted which concluded that the external locus of control had a effect on the general well beingwhere as the internal locus of control doesn't have any impact. [8] This can be related to the results obtained as there was high difference between the two.

Along with locus of control, gender also is seen to play a significant role in the general well being of the students. The mean general well being of male participants was 68.15 and female was 60.68 . The interpretation of this is that males have a higher general well-being than females.

These findings can be explained by the following theories:

\section{Gender Socialisation}

Socialisation is the process of internalising society's values in order to adapt to one's culture .It influences how people behave as males and females in society. The social learning process that imbibes people into understanding the various aspects of culture includes the process of gender socialisation.

Indian society is marked by deep gender inequality, there is a difference in the family experience of the Indian youth based on their gender, the independence enjoyed also varies. Childhood experiences, self esteem and degree of freedom influences one's mental health, thus the gender of an individual directly impacts well-being.

\section{Flourishing}

According to Corey Keyes and Carol Ryff, mental well-being has three components, namely emotional/subjective, psychological and social well-being. Emotional well-being concerns subjective aspects of well-being, i.e. feeling well, whereas psychological well-being concerns skills, abilities, and social well-being talks about social functioning.

The researchers came to the conclusion that females are low on all the three components of well-being. This is due to several factors, mainly the amount of importance and encouragement to give male sportsmen as compared to their female counterparts. This makes the struggle for female players worse, they not only have to compete with other female players but also prove themselves at par with the male players in the same sport [9]. Thus, gender has a greater impact on the general well-being score and the impact of sport participation is not significant.

\section{Ancillary Observation}

1. The graphs indicate the distribution of the 7 dimensions of general well-being, i.e., Positive Wellbeing, Low Positive, Marginal, Stress Problem, Distressed, Serious and Severe for male sports, male non sports, female sports and female non sports. It can be observed that, male non sport participants have the highest percentage of people with positive well-being score whereas, female sport participants have the lowest percentage of people with positive well-being score.

2. The graphs tell us that female participants have high scores lying between serious and severe dimensions, female non sport participants having a higher percentage of people with a very low well-being score.

3. In the total sample we observed that the highest number of people lie under the dimension of stress problem.

4. It was observed that, a larger percentage of sport participants had an internal locus of control as compared to non participants. $80 \%$ of the sport participants i.e., 64 participants had an internal locus of control and $73.75 \%$ - non-participants i.e., 59 participants had an internal locus of control. 


\section{Limitations and Implications}

1. The researcher used a convenience and purposive sample and not a randomised one.

2. The sample set size of the researcher's survey was 42 items in all which was too lengthy, this may have led to fatigue effect and lower quality of responses.

3. The high face validity might have led to respondents desire to improve their self-image.

4. The researcher did not control the environmental or situational factors that might have led to a difference in response, this was because digital communication was used to distribute the survey i.e. google forms.

5. Powerful others and chance were categorised as determinants of external locus of control, and not independent variables.

6. Sample size between students who have internal locus of control and external locus of control is different.

7. In further studies, the researcher suggests that one can take specific sports

\section{CONCLUSIONS}

It can be concluded that locus of control and gender plays a significant role in the psychological well being, this is contradictory to what the researchers hypothesised. Sport in the researcher's sample, did not have major impact on the general well-being of students. The researcher's study, was in line with previous researches, saying that gender has a major impact on general well-being of an individual, this is because of several psycho-social factors.

\section{REFERENCES}

1. Nwankwo BC, Okechi BC, Kalu OE. Role of Locus of Control and Gender on Psychological Well-being among Youth Athletes. Journal of Psychology and Sociological Studies 2017;1(1).

2. Kleiber DA, Hemmer JD. Sex differences in the relationship of locus of control and recreational sport participation. Sex Roles 1981;7(8):801-10.

3. Malebo A, Van Eeden C, Wissing MP. Sport participation, psychological well-being, and psychosocial development in a group of young black adults. South Afr J Psychol 2007;37(1):188-206.

4. Kavetsos G, Szymanski S. National well-being and international sports events. J Econ Psychol 2010;31(2):158-71

5. Mirzaei A, Nikbakhsh R, Sharififar F. The relationship between personality traits and sport performance. Eur J Experiment Biol 2013;3(3):439-42.

6. Keyes CL. The mental health continuum: From languishing to flourishing in life. J Health Soc Behav 2002;6:207-22.

7. Parati G, Omboni S, Compare A, Grossi E, Callus E, Venco A, Destro M, Villa G, Palatini P, Rosei EA, Scalvini S. Blood pressure control and treatment adherence in hypertensive patients with metabolic syndrome: protocol of a randomized controlled study based on home blood pressure telemonitoring vs. conventional management and assessment of psychological determinants of adherence (TELEBPMET Study). Trials 2013;14(1):22-8.

8. Lefcourt HM, editor. Locus of control: Current trends in theory \& research. Psychology Press; 2014.

9. Mohanty J. Modern trends in Indian education. Deep and Deep Publications; 2004

$* * * * * * * * * * * * * * * * * *$

Acknowledgements - Nil

Conflict of Interest - Nil

Funding - Nil 